\title{
New Results in Nucleon-Nucleon Scattering at Intermediate Energies
}

\author{
H. Spinka \\ High Energy Physics Division \\ Argonne National Laboratory, Argonne, Ilinois 60439, USA
}

\begin{abstract}
Many np elastic scattering spin observables have recently been measured between kinetic energies of about 500 and $1100 \mathrm{MeV}$ at Saclay and LAMPF. These data are summarized and some new results are presented. Evidence for structure in pp observables near $2100 \mathrm{MeV}$ is reviewed, and new data in this energy region are shown from SATURNE.
\end{abstract}

\section{A) INTRODUCTION}

Nucleon-nucleon (N-N) interactions have had a significant impact on a wide variety of topics in nuclear and particle physics. These include the understanding of the nuclear force, nucleon scattering from nuclei, and the allowable quark configurations, such as perhaps 6-quark states.

Nucleon-nucleon elastic scattering is one of the most basic reactions involving the strong interaction. The $\mathrm{N}-\mathrm{N}$ elastic amplitudes for many years have served as stringent constraints on theoretical models describing the nuclear force at intermediate energies. Such models have included meson exchange, Skyrme, and QCD-inspired parton models. The differences in inelastic channels for the isospin-0 $(I=0)$ and isospin-1 $(I=1) \mathrm{N}-\mathrm{N}$ interactions have been significant in the development of some of these models. The nucleon-nucleon reactions are becoming important for the study of spin effects of the nucleon constitutents, since the nucleon contains relatively few valence quarks, and since such spin effects are partially masked in mesonnucleon amplitudes by the zero spin of both pions and kaons.

Measurements of $\mathrm{N}-\mathrm{N}$ interactions have been useful for calculations of the scattering of nucleons from nuclei [1]. At present, these calculations either directly use the free $\mathrm{N}-\mathrm{N}$ amplitudes from phase shift analyses of the experimental data [2-5], or use the results of theoretical models, fit to the $\mathrm{N}-\mathrm{N}$ amplitudes, to predict the effective nucleon-nucleon interaction inside nuclei. In the future, one goal will be to understand nucleon scattering from nuclei in terms of quark and gluon interactions. Free $\mathrm{N}-\mathrm{N}$ scattering will provide a stringent test case in the work to achieve this goal. 


\section{DISCLAIMER}

This report was prepared as an account of work sponsored by an agency of the United States Government. Neither the United States Government nor any agency thereof, nor any of their employees, makes any warranty, express or implied, or assumes any legal liability or responsibility for the accuracy, completeness, or usefulness of any information, apparatus, product, or process disclosed, or represents that its use would not infringe privately owned rights. Reference herein to any specific commercial product, process, or service by trade name, trademark, manufacturer, or otherwise does not necessarily constitute or imply its endorsement, recommendation, or favoring by the United States Government or any agency thereof. The views and opinions of authors expressed herein do not necessarily state or reflect those of the United States Government or any agency thereof. 


\section{DISCLAIMER}

Portions of this document may be illegible in electronic image products. Images are produced from the best available original document. 
The third motivation for N-N spin measurements concerns the observation of energy dependent structures seen in various reactions and spin parameters. For example, there has been considerable controversy [6-13] about the interpretation of the resonance-like behavior of the ${ }^{1} \mathrm{D}_{2}$ and ${ }^{3} \mathrm{~F}_{3}$ partial waves seen in phase shift analyses. Evidence from a number of experiments has also suggested structure near a kinetic energy of $2100 \mathrm{MeV}$ in pp reactions. This energy is well above the thresholds for production of low-lying $N^{*}, \Delta$, and $\Lambda$ states. It will be important to try to confirm these structures, and to understand their origin, since 6-quark states are predicted near this energy; see Sec. C.

Assuming the usual symmetries for the strong interaction, there are five $I=0$ and five $I=1$ nucleon-nucleon elastic scattering amplitudes at each angle and energy. Therefore, at least ten spin observables must be measured for pp scattering and ten additional observables for np scattering to obtain unique values for both the real and imaginary parts of these amplitudes. Note that $\mathrm{pp}$ and $\mathrm{nn}$ scattering are pure $\mathrm{I}=1$ channels, whereas the $\mathrm{np}$ scattering amplitudes are mixtures of $I=0$ and $I=1$ channels,

$$
A m p l(n p)=\frac{1}{2}[\operatorname{Ampl}(I=0)+A m p l(I=1)],
$$

at each angle and energy. The factor of $1 / 2$ in the equation above indicates that the $I=0$ amplitudes will generally be more poorly determined than the $\mathrm{I}=1$ amplitudes, assuming that the $\mathrm{pp}$ and $\mathrm{np}$ spin observables are measured to the same accuracy. On the other hand, np measurements are more difficult because polarized neutron beams are usually of lower intensity and have a broader energy spread than proton beams, neutron counters normally have significantly less than $100 \%$ efficiency and poorer spatial resolution than charged particle detectors, etc.

At intermediate energies, a large amount of new data in the 1980's on pp spin observables has permitted a reasonable determination of the $I=1$ amplitudes in terms of phase shifts and inelasticities up to $\sim 1700 \mathrm{MeV}$. In addition, the pp elastic scattering amplitudes have been found in a modelindependent fashion over part of the scattering angle range at 19 energies up to $T_{\text {lab }}=5.14 \mathrm{GeV}$ or $P_{1 a b}=6.00 \mathrm{GeV} / \mathrm{c}$. This includes: a) model independent amplitude analyses [14-16] up to $800 \mathrm{MeV}$ that have been performed and published at 7 energies, b) a determination [17] of the pp amplitudes at 11 somewhat coarsely-spaced energies between 830 and $2700 \mathrm{MeV}$ by the Saclay nucleon-nucleon collaboration, and c) a model independent amplitude determination [18] and phase shift analysis [19] that were performed at the single energy of $5.14 \mathrm{GeV}$. By contrast, many fewer $\mathrm{I}=0$ model independent amplitude determinations have been performed, and the number of angles is small compared to the $I=1$ case. 
The spin observables in this paper will be described in terms of the spin directions shown in Fig. 1. The directions are $\vec{N}$, normal to the scattering plane, $\vec{L}$, longitudinal along the beam or outgoing particle directions, and $\vec{S}=\vec{N} \times \vec{L}$ in the scattering plane. The observables will be denoted $(B, T ; S, R)$ with $B$ the beam, $T$ the target, $S$ the forward scattered, and $R$ the recoil spin direction, and " 0 " denotes that the spin direction is not measured or is unpolarized. Other notations [20] will also be used to assist the reader to translate between the various expressions used by different groups for the same spin observables.

FIGURE 1. Definition of the spin directions for the beam, target, forward-scattered, and recoil particles for nucleon-nucleon elastic scattering.

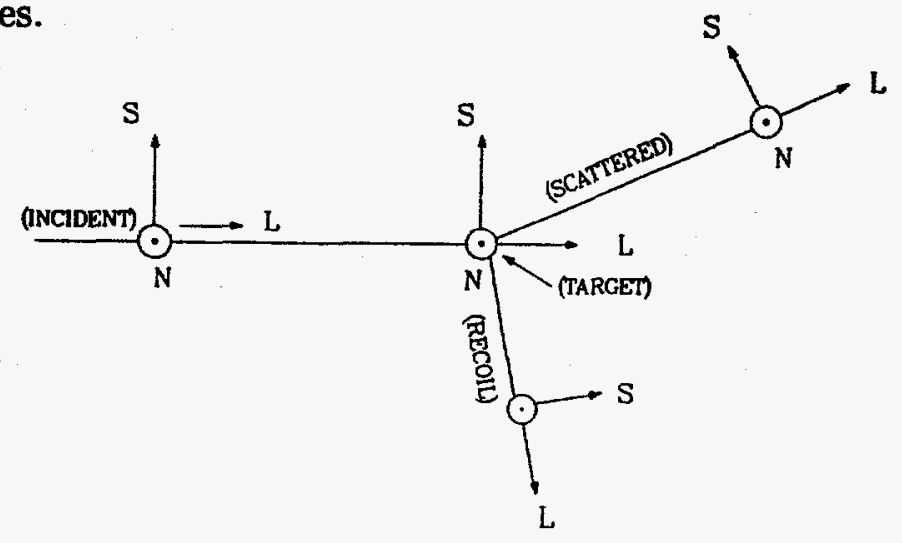

N: NORMAL, TO THE SCATTERING PLANE

L. LONGTTUDINAL DIRECTION

$\mathrm{S}=\mathrm{N} \times \mathrm{L}$ IN THE SCATTERJNG PLANE

\section{B) np SCATTERING AT LAMPF AND SATURNE}

Several years ago, a number of authors $[21,22]$ noted the poor state of knowledge of $I=0$ nucleon-nucleon elastic amplitudes or phase shifts above a kinetic energy of $\sim 500 \mathrm{MeV}$. Problems were also indicated $[23,24]$ in inelastic $\mathrm{I}=0$ cross sections.

Within the past few years, a huge amount of new np elastic and total cross section spin data have been published from extensive programs at Los Alamos and Saclay. The collaborating institutions at SATURNE included the DAPNIA and LNS departments, CE-Saclay, France; DPNC, University of Geneva, Switzerland; Freiburg University, Germany; LNP-JINR, Dubna, Russia; University of California at Los Angeles; C.E.N.B., Gradignan, France; and the HEP division, Argonne National Laboratory. Collaborators on the LAMPF experiments included physicists from LAMPF; University of Central Arkansas; University of Colorado; Earlham College; University of Montana; New Mexico State University; Rice University; Rudjer Boskovic Institute in Zagreb, Croatia; Rutgers University; Texas A\&M University; University of Texas at Austin; Washington State University; University of Wuppertal, Germany; and the HEP division, Argonne National Laboratory. 
The new LAMPF data were collected in three different experimental arrangements at the energies 484, 567, 634, 720, and $788 \mathrm{MeV}$. Polarized neutrons were produced by forward charge exchange of polarized protons on a liquid deuterium target. One set of experiments used the new high intensity optically pumped polarized ion source, and consisted of a liquid hydrogen target, a magnetic spectrometer and carbon rescattering polarimeter for the outgoing proton [25], and a neutron counter array [26]. These neutron counters were only used to provide a check on the results, and especially the background subtraction. The measured np spin observables included $P=A_{N 0}=A_{\text {oono }}=(N, 0 ; 0,0), K_{N N}, K_{S S}, K_{L S}, K_{S L}$, and $K_{L L}=K_{o k^{\prime \prime} k_{o}}=(L, 0 ; 0, L)$ [27-29]; only three of the last four observables are independent. These experiments also permitted an improved measurement of the neutron beam polarization [30], which deviated by $\sim 12 \%$ from previous estimates. Some data recently collected on $P$ and $K_{N N}$ over a broader angular range than in [29] are presently being analyzed.

A second setup used beam from the old, lower intensity Lamb-shift polarized ion source, and consisted of a polarized proton target and a large aperture magnetic spectrometer. The polarized target magnet prevented the simultaneous detection of neutrons. The measured spin observables included $C_{S S}, C_{L S}=C_{S L}$, and $C_{L L}=A_{\text {ookk }}=(L, L ; 0,0)$. All the elastic scattering data from these experiments are analyzed, including corrections for the new beam polarization measurements, and are shown in Fig. 2. Some of the data have been published [31,32], and a model independent $\mathrm{I}=0$ amplitude determination is planned using the LAMPF data at $\sim 484,634$, and 788 $\mathrm{MeV}$.

The third setup was nearly the same as the second, except that the magnetic spectrometer was replaced with a set of neutron counters [26] in the beam at $0^{\circ}$. The total cross section difference between antiparallel and parallel longitudinally polarized neutron beam and proton target, $\Delta \sigma_{L}(n p)=$ $\sigma_{\text {tot }}(\stackrel{\leftrightarrow}{\rightarrow})-\sigma_{\text {tot }}(\rightarrow \rightarrow)$, was measured at five energies. The results were recently published [33] and are shown in Fig. 3.

The majority of the new SATURNE data were collected using a free polarized neutron beam produced by stripping polarized deuterons. The neutron beam struck a frozen spin polarized proton target. Outgoing protons were measured with scintillation counters and wire chambers, and outgoing neutrons were detected either in a neutron counter array or in a pair of wire chambers after conversion in a carbon block [34]. Either the polarization of the outgoing protons was determined by rescattering in a carbon polarimeter, or the proton momentum was measured in a magnetic spectrometer for these results.

A large number of spin parameters were measured at energies of 800 , $840,860,880,910,940,1000,1080$, and $1100 \mathrm{MeV}$ [35-39]. These included 

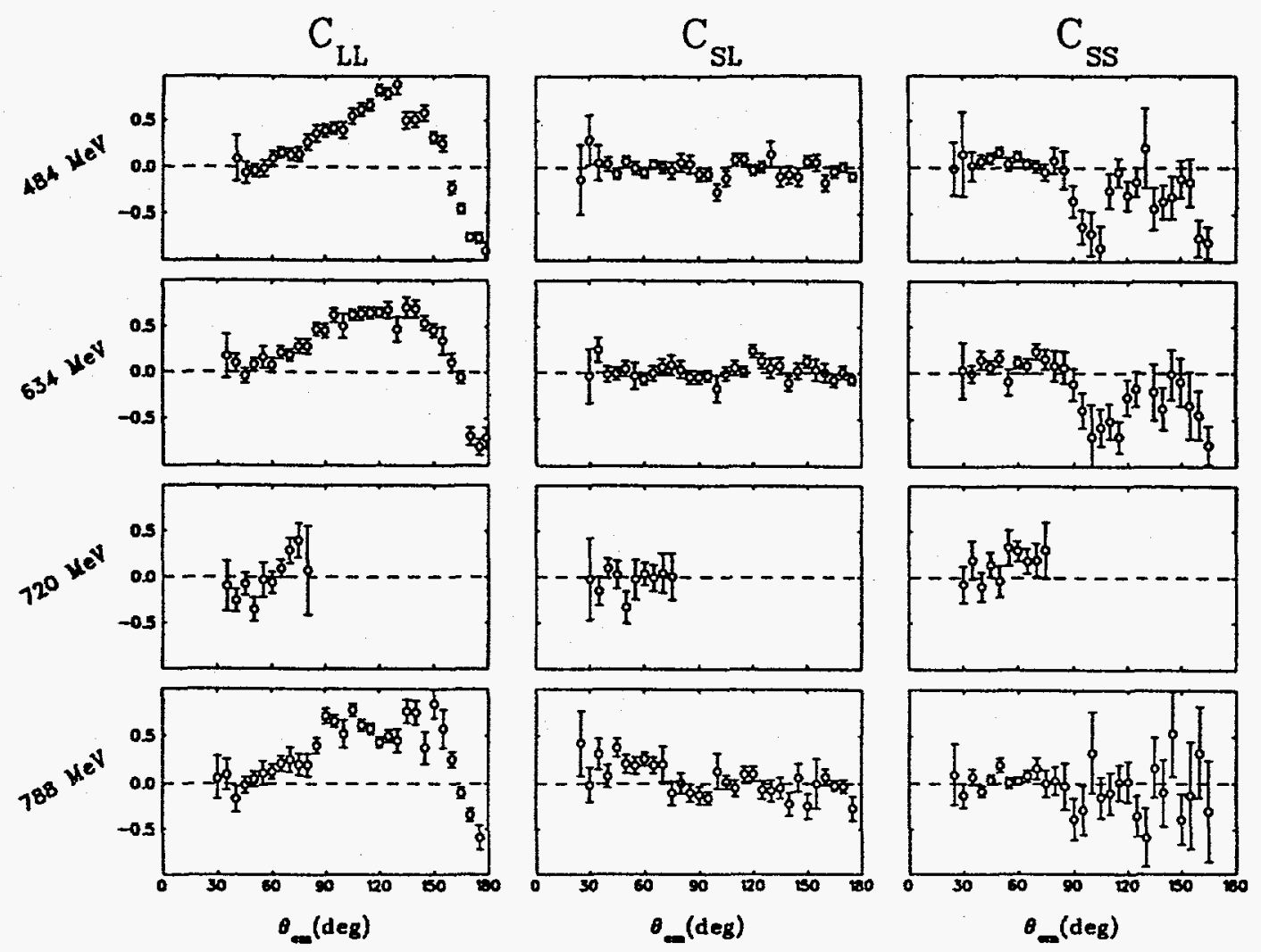

FIGURE 2. Final values for the spin parameters $C_{S S}=(\mathrm{S}, \mathrm{S} ; 0,0), C_{L S}$, and $C_{L L}$ with a polarized neutron beam on a polarized proton target at LAMPF. Some of these results were extracted from measurements of mixtures of the observables.

$P, C_{N N}, C_{S L}, C_{L L}, D_{N N}, D_{L S}=D_{o s " o k}=(0, L ; 0, S), K_{N N}, K_{L S}, K_{S S}$, $H_{S L N}=N_{\text {onsk }}=(S, L ; 0, N)$, and $H_{L L N}$. A model independent amplitude determination [40] is in progress using these data.

The total cross section differences $\Delta \sigma_{L}(n p)$ and $\Delta \sigma_{T}(n p)$ (which is defined analogously for transverse spins) were measured [41,42] using nearly identical sets of counters before and after the polarized target instead of the charged particle and neutron detectors used for the elastic scattering measurements. The $\Delta \sigma_{L}(n p)$ results from Saclay and from PSI [43] are shown in Fig. 3. Good agreement among the three data sets can be seen. The $\mathrm{I}=0$ cross sections can be obtained from $\Delta \sigma_{L}(I=0)=2 \Delta \sigma_{L}(n p)-\Delta \sigma_{L}(p p)$ and similarly for $\Delta \sigma_{T}$; see Fig. 4. These expressions follow from the generalized optical theorem, where $\Delta \sigma$ is proportional to the imaginary part of a forward elastic amplitude. It is surprising that the size of the energy dependent structure seen in $\Delta \sigma_{L}(I=0)$ is comparable to that for $\Delta \sigma_{L}(I=1)$, even though the strong $N N \rightarrow N \Delta$ channel cannot contribute to $I=0$, and the inelastic $\mathrm{I}=0$ cross section is much smaller than the $\mathrm{I}=1$ inelastic cross section at these energies. A possible interpretation in terms of a dibaryon state is described in [33]. 


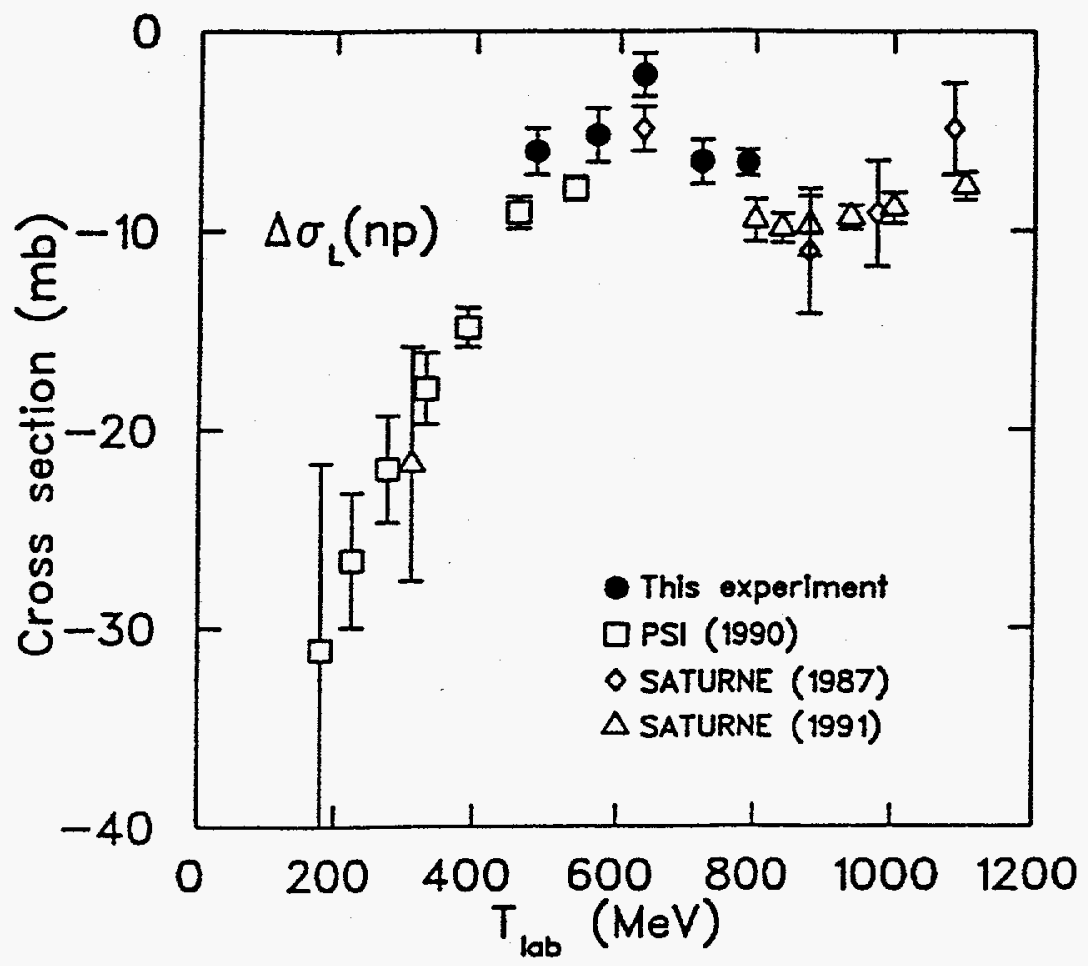

FIGURE 3. Total cross section difference in longitudinal spin states for $\mathbf{n p}$ interactions from $[33,41,43]$. In all cases, a free polarized neutron beam and a polarized proton target were used.
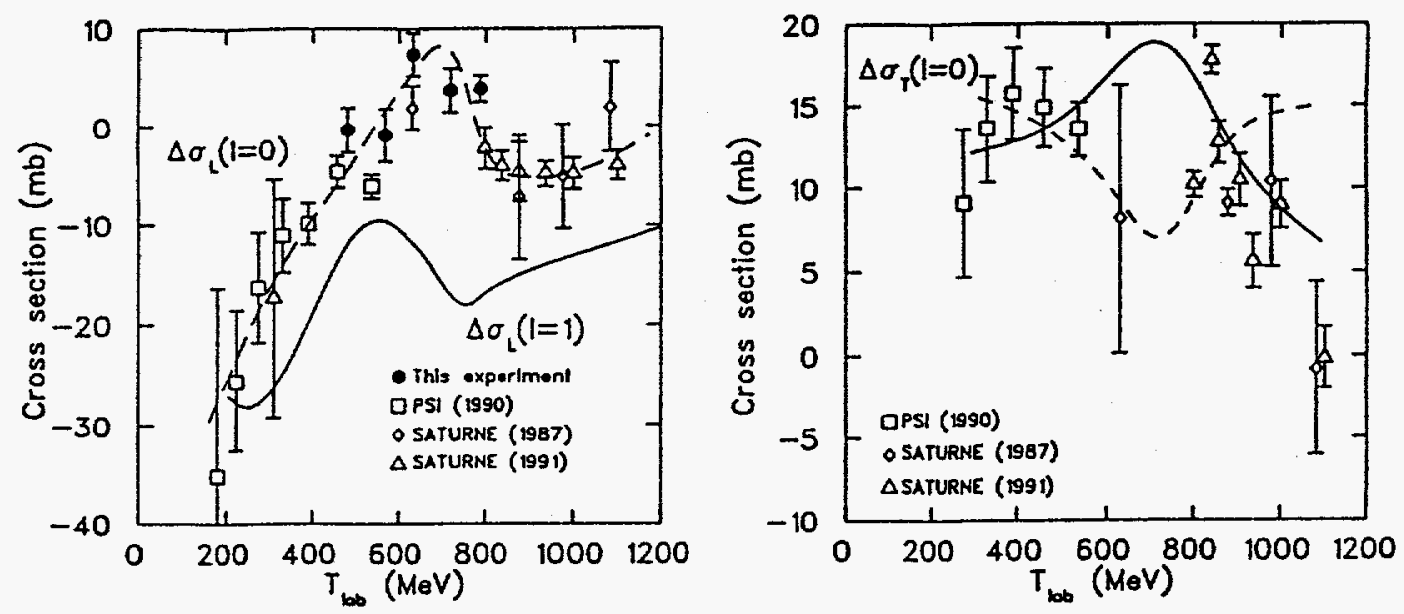

FIGURE 4. Total $I=0$ cross section differences in longitudinal and transverse spin states obtained from pp and np measurements in Los Alamos, Saclay, and other laboratories. The left figure shows $\Delta \sigma_{L}(I=0)$ data. The dashed curve is drawn to guide the eye, whereas the solid curve is drawn through the $\mathrm{I}=1$ data. The right figure presents the $\Delta \sigma_{T}(I=0)$ results, and the two curves are from models in [33]. 
The improvement in the np data base can be illustrated by plotting the total number of data points with statistical errors up to some size as a function of that size. The very numerous, and sometimes conflicting, differential cross section and polarization parameter measurements are excluded. The results for kinetic energies between 400 and $900 \mathrm{MeV}$ for pp and np spin observables up to 1984, for $\mathrm{np}$ data up to 1994 , and for $\mathrm{np}$ data between 400 and $1100 \mathrm{MeV}$ in 1994 are shown in Fig. 5. The impressive increase in new data is immediately apparent for the whole range of statistical errors.

Additional np measurements are in progress at TRIUMF [44], and a major new program is under way at PSI [45]. In addition, pp elastic scattering experiments are being performed at the Indiana cooler [46], and are being prepared for COSY.

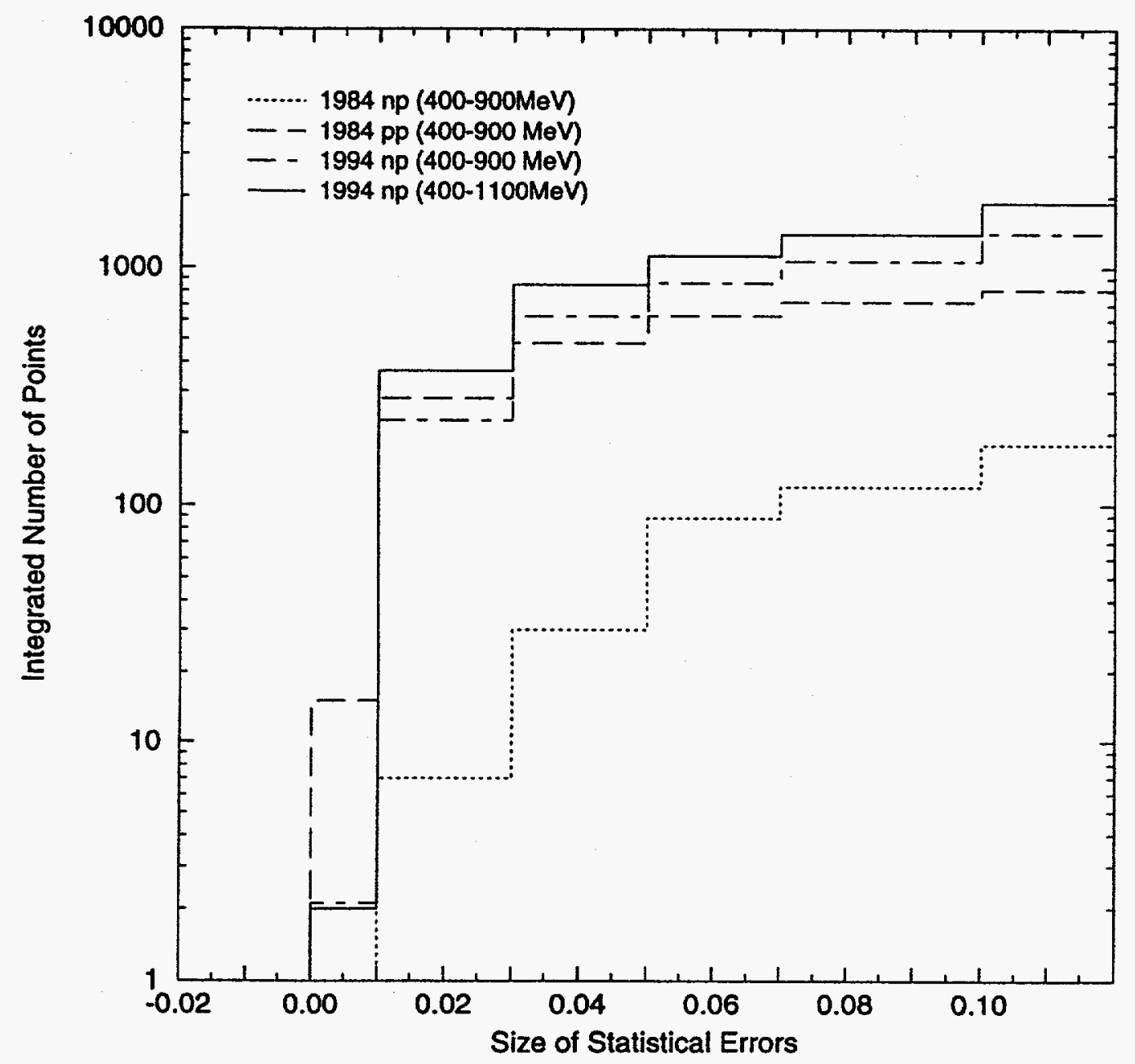

FIGURE 5. Integrated number of data points as a function of the statistical error for nucleon-nucleon elastic scattering spin observables except the polarization parameter and the differential cross section. 
Knowledge of the $I=0$ nucleon-nucleon phase shifts has increased substantially over the past few years. The agreement between various phase shift analyses is expected to improve as the new np data are incorporated into the data bases for these analyses.

\section{C) pp SCATTERING AT SATURNE}

A number of early theoretical approaches suggested that dibaryon states should exist. These included models based on SU(3) and SU(6) symmetries $[47,48]$ and on Regge ideas [49,50]. A variety of experimental evidence appeared to support these theories, such as a "bump" in the pp total cross section [51] and breaks in the slope of the $90^{\circ} \mathrm{pp}$ differential cross section $[52,53]$. Later, theoretical arguments were presented, based on Regge ideas and duality, that the N-N channel is "exotic" and without resonances [54]. As a consequence, the physics community strongly believed there were no dibaryons except deuteron-like objects. This belief was strengthened by the fact that the interpretation of many experimental data in terms of dibaryon states was often ambiguous. For example, energy dependent structure was often near strong inelastic thresholds, or of insufficient statistics, or nonreproducible, etc.

More recently, QCD-inspired bag and cloudy-bag models [55-58], constituent quark models [59,60], and diquark cluster models [61] have appeared which also predict dibaryon states. The masses for the expected lowest lying

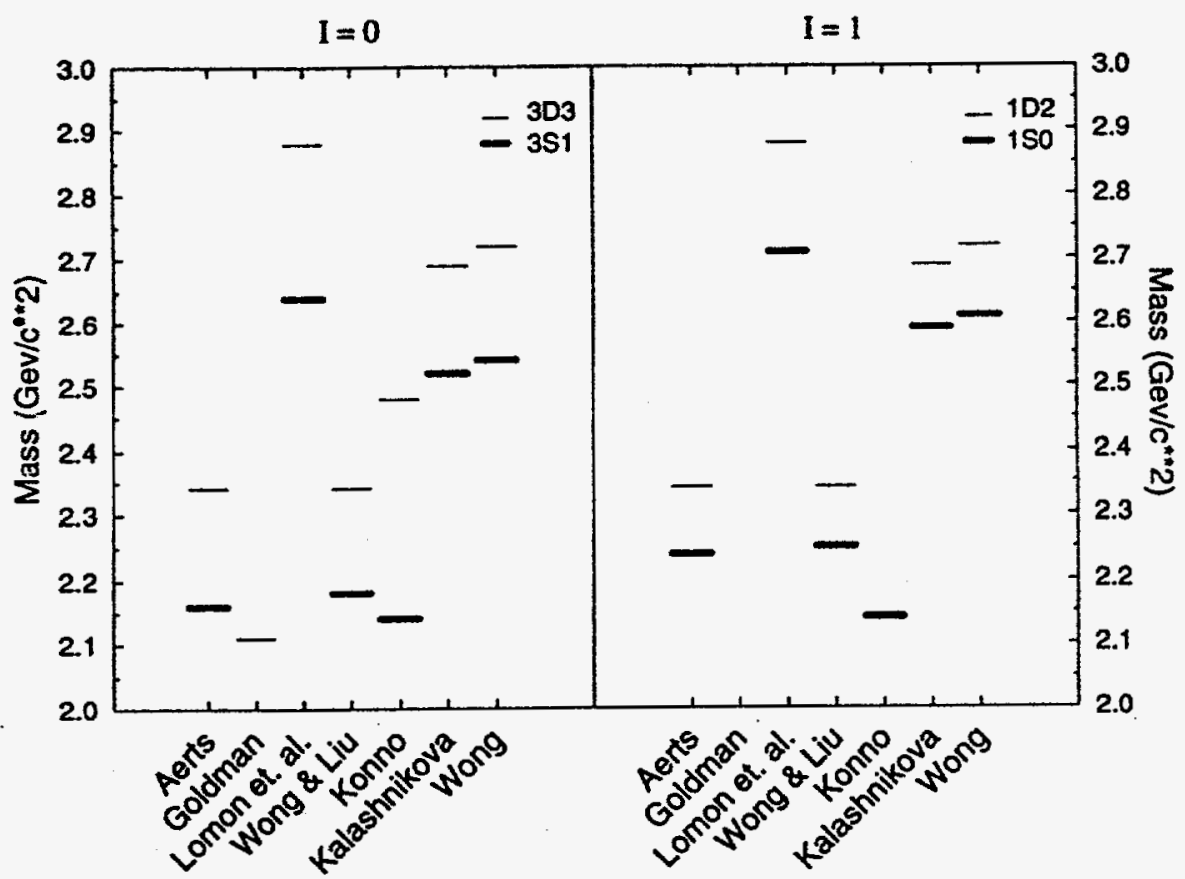

FIGURE 6. Predicted masses for the ${ }^{3} S_{1},{ }^{3} D_{3},{ }^{1} S_{0}$, and ${ }^{1} D_{2}$ dibaryon states in various quark models [55,57-61]. Generally, these are the lowest lying states in the models. 
states differ for these models; see Fig. 6 . Several of the recent models predict the lowest $\mathrm{I}=1$ dibaryon states to have masses above $2.4 \mathrm{GeV}$.

It seems that a whole range of QCD-inspired models are able to predict 6-quark states, while there appear to be none that forbid dibaryons. If 6-quark states do not exist, then there is probably something wrong or missing from the present models. The missing ingredient could have extremely important consequences for the understanding of nuclear matter. Therefore, further experiments are needed to clarify whether dibaryons exist.

For dibaryon masses above about $2.4 \mathrm{GeV}$, there are many inelastic thresholds present, and the widths of $\mathrm{N}^{*}$ and $\Delta$ states cause many of these thresholds to effectively overlap. As noted before, phase shift analyses are not reliable in this energy range at this time. Yet, many energy dependent effects are observed in a variety of reactions; see Fig. 7. For example, the pp total cross section data indicate a bump [51], polarization measurements at the 4 -momentum transfer squared $\mathrm{u}=0$ in the $\mathrm{pp} \rightarrow \pi \mathrm{d}$ reaction exhibit large changes [62,63], and the Saclay pp amplitude determinations [17] show. a sizeable variation at $T_{\text {lab }}=2100 \mathrm{MeV}$, compared to 1800 and $2400 \mathrm{MeV}$. For this last case, $C_{S L}=-A_{x z}=A_{\text {oosk }}=(\mathrm{S}, \mathrm{L} ; 0,0)$ data near $\theta_{c m}=45^{\circ}$ from Saclay $[64,65]$ and the ZGS [66] are shown in Fig. 7. There is also a suggestion of structure in $\Delta \sigma_{L}(p p)$ from ZGS data $[67,68]$.

Recently, measurements were performed of the spin observable $C_{N N}$ in pp elastic scattering at a set of 14 finely spaced energies at Saclay [69]. These results show a sharp drop in $C_{N N}\left(90^{\circ}\right)$ with energy. Predictions of this spin observable by González, LaFrance, and Lomon in their cloudy bag model [58] appear reasonably consistent with the observations. This model has been quite successful in reproducing the pp phase shifts at lower energies.

The drop in $C_{N N}=A_{\text {oonn }}$ at $\theta_{c m}=90^{\circ}$ may be related to changes in spin singlet wave(s), since at that angle,

$$
\left(1-A_{\text {oonn }}\right) \frac{d \sigma}{d \Omega} \sim \mid \text { Singlet Ampl. }\left.\right|^{2} .
$$

Thus, if a singlet wave becomes quite strong or resonates, $A_{\text {oonn }}$ would decrease. At this time it is unclear whether the changes seen in the direct amplitude reconstruction [17] or in the polarization for $\mathrm{pp} \rightarrow \pi \mathrm{d}[62,63]$ are related to the behavior of spin singlet wave(s). However, an increase in the strength of such waves at $\theta_{c m}=0^{\circ}$ would appear as a peak in $\Delta \sigma_{L}(p p)$, as is suggested by the data in $[67,68]$.

Two recent runs at Saclay by the N-N collaboration were devoted to rechecking the earlier results in [69] and to extending them in energy. The runs in November and December, 1993 measured a variety of two and three spin observables at $\mathrm{T}_{\text {lab }}=1800$ and $2100 \mathrm{MeV}$ to clarify the behavior of the pp amplitudes reported earlier in [17]. Included were new data for $P$ and $C_{N N}$ at these energies and at 1850 and $2040 \mathrm{MeV}$. The runs in May and 


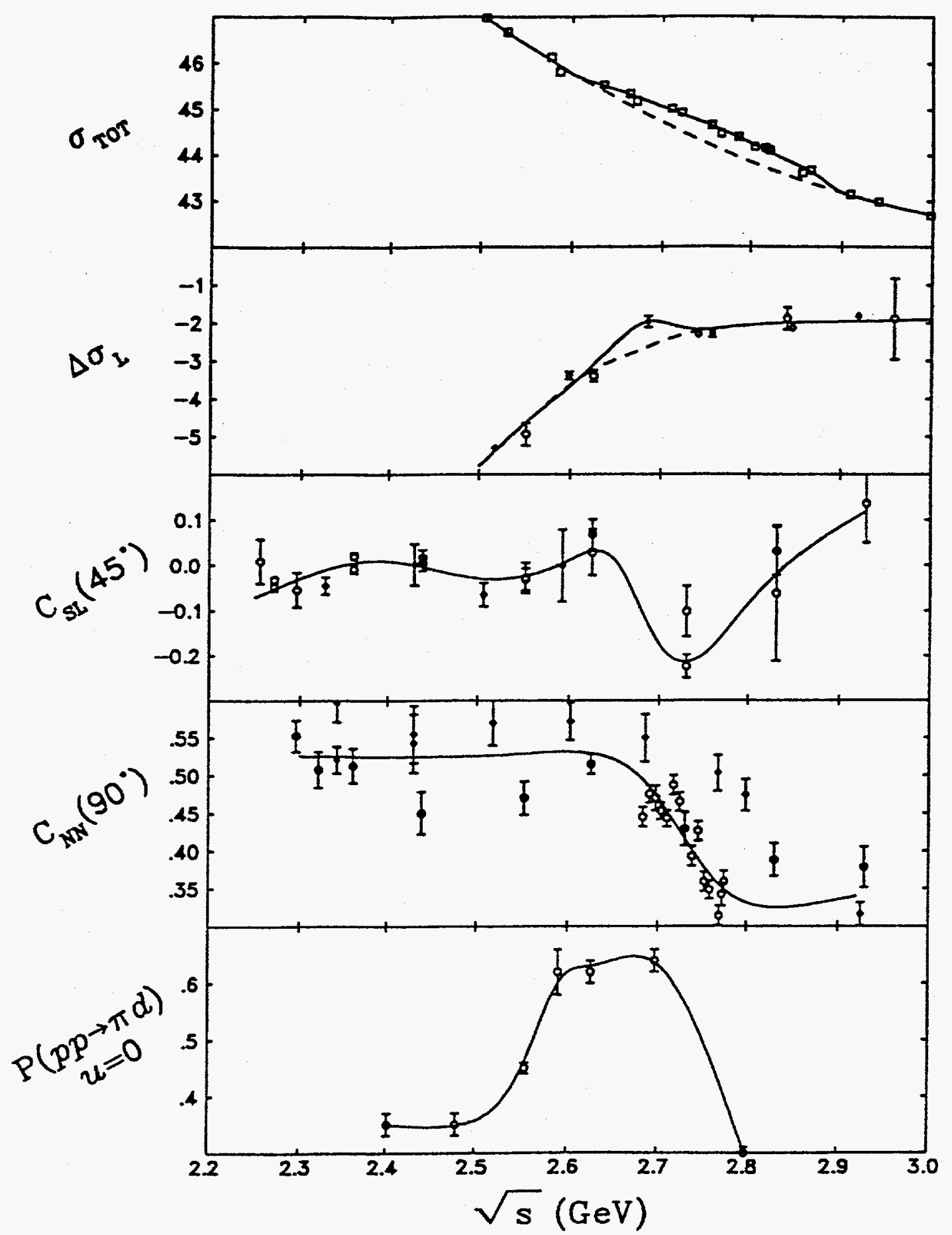

FIGURE 7. Collection of various data showing energy dependent behavior near $\sqrt{s}=$ 2.7 GeV. The pp total cross section data are from [51], $\Delta \sigma_{L}(p p)$ from $[67,68]$, the pp elastic scattering spin observables $C_{S L}$ and $C_{N N}$ from [64-66] and [69-72] respectively, and polarization at $u=0$ for the $p p \rightarrow \pi d$ reaction from [62,63]. All curves are drawn to guide the eye. 
June, 1994 measured $P$ and $C_{N N}$ as well, rechecking several energies reported in [69] and extending the data in coarse steps up to $2650 \mathrm{MeV}$. If the structure seen near $\mathrm{T}_{\text {lab }}=2100 \mathrm{MeV}$ is caused by a ${ }^{1} \mathrm{~S}_{0}$ dibaryon (see Fig. 6), then the ${ }^{1} \mathrm{D}_{2}$ state is expected near $2500-2600 \mathrm{MeV}$, and another drop in $C_{N N}$ should be seen.

A number of experimental improvements were made in these two runs. Additional counters and electronics for monitoring beam polarization and intensity were employed. A new material was used for the polarized target which gave a faster polarizing time. A new data acquisition system was added; this system has improved capabilities to monitor the hardware performance. Checks of the incident beam angle were performed at a number of energies with the polarized target magnetic field turned off. The target polarization direction was reversed between each energy change, and data were recorded with "zero" beam polarization state at each energy. These changes should allow better control of systematic errors compared to the earlier data.

A preliminary analysis of data at a few energies has been performed, but not all systematics checks have been included. The data are normalized to the same beam polarimeter analyzing power as the published data [69]. The November, 1993 and May, 1994 preliminary results agree well with the earlier measurements at $2040 \mathrm{MeV}$ as seen in Fig. 8; data at $2120 \mathrm{MeV}$ agree similarly. Somewhat poorer agreement is observed at $2220 \mathrm{MeV}$ (Fig. 9) and $2160 \mathrm{MeV}$. The origin of the differences is not understood at this time.

Preliminary results for $C_{N N}\left(90^{\circ}\right)$ are given in Fig. 10 as well as published results from $[69,70]$. Although the agreement in Figs. 8-10 appears quite good, it is premature to conclude that the energy dependent structure is confirmed until all the new checks of systematic effects have been carefully studied. A reanalysis of the data in [69] may also be required. In any case, it is anticipated that the new measurements will clarify the past Saclay observations on structure in this energy region.

\section{D) CONCLUSIONS}

A large amount of new np elastic scattering and total cross section spin data have recently been analyzed. This includes about 850 new points between 480 and $790 \mathrm{MeV}$ from LAMPF and 1600 new points between 470 and $1100 \mathrm{MeV}$ from SATURNE in the past four years (or about 760 and 950 points excluding $P$ ). These new results will permit model independent $\mathrm{I}=0$ amplitude determinations for the first time at 8 energies and a number of scattering angles. Phase shift analyses for $\mathrm{I}=0$ partial waves will also be greatly improved.

Several different spin observables and reactions suggest energy dependent structure near $\sqrt{s}=2.7 \mathrm{GeV}$ or $\mathrm{T}_{\text {lab }} \sim 2100 \mathrm{MeV}$ in pp elastic scattering; see Fig. 7. For example, a sizeable change in the pp elastic amplitudes 


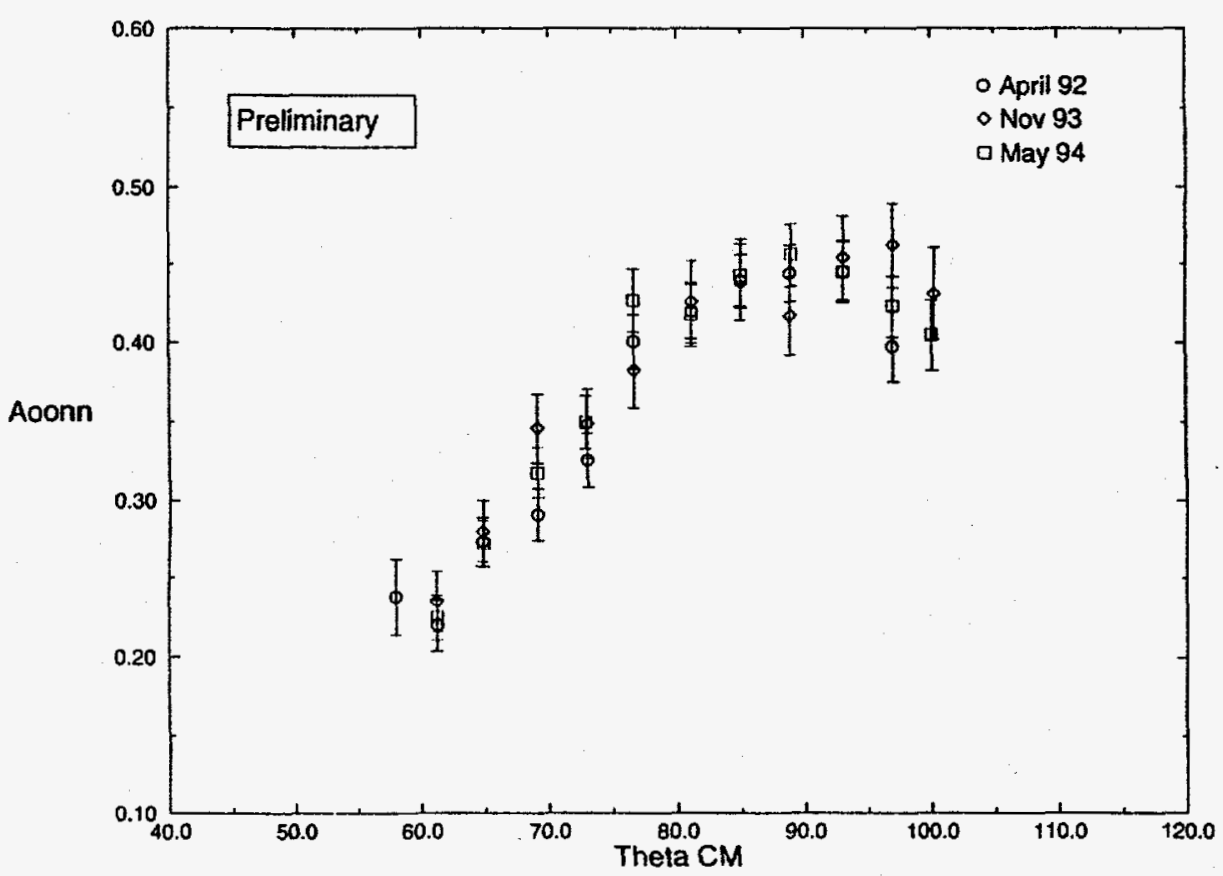

FIGURE 8. Comparison of $C_{N N}$ data for pp elastic scattering at $2040 \mathrm{MeV}$ collected in different runs as a function of angle. The Nov., 1993 and May, 1994 results are preliminary.

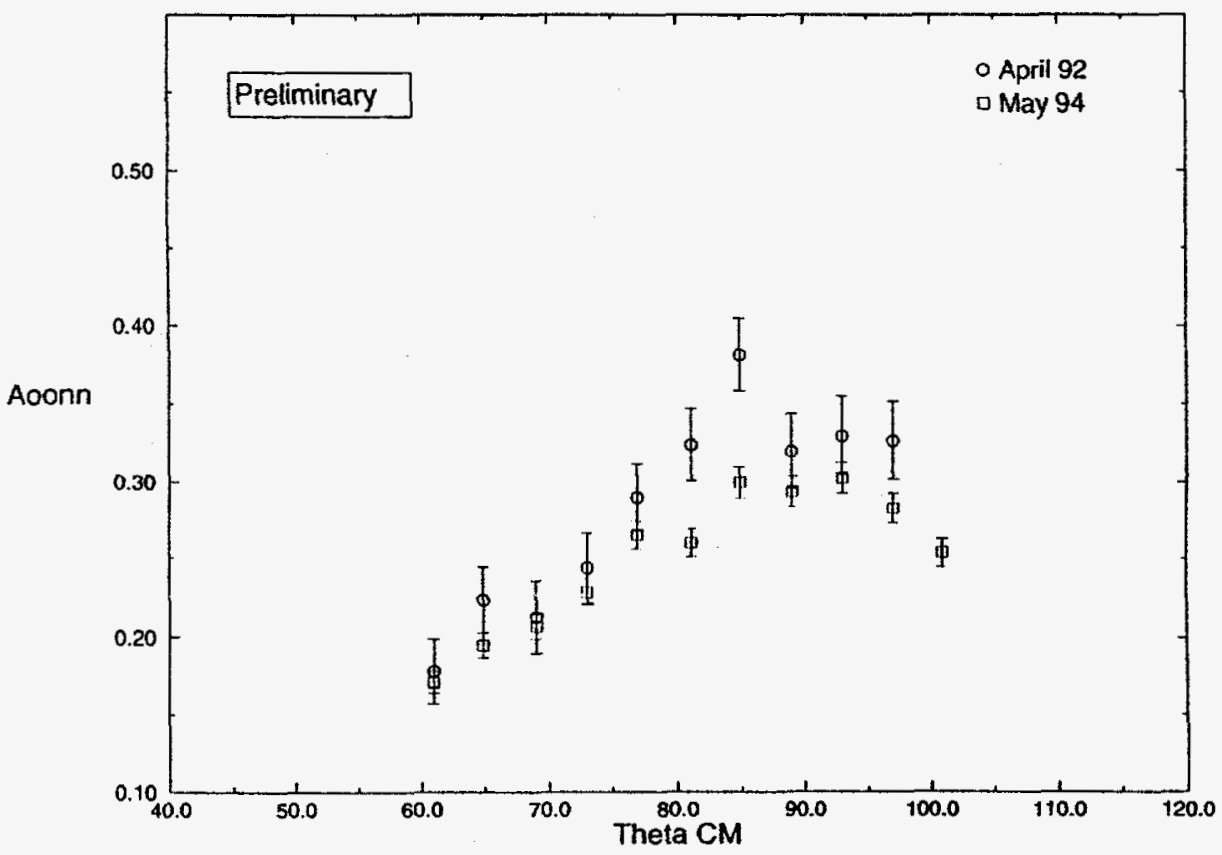

FIGURE 9. Comparison of $C_{N N}$ data for pp elastic scattering at $2220 \mathrm{MeV}$ collected in different runs as a function of angle. The May, 1994 results are preliminary.

between 1800,2100 , and $2400 \mathrm{MeV}$ was observed in a model independent amplitude reconstruction [17]. Additional data were collected at the first two beam energies to clarify these results. Another example is the drop in 
the pp spin observable $C_{N N}\left(90^{\circ}\right)$ seen near $2100 \mathrm{MeV}$ at SATURNE [69]. Several energies have been repeated and the energy range extended in recent experiments. Using certain assumptions, the new and published $C_{N N}$ results seem to agree reasonably well, but many checks of systematic effects are still required of the new data. Once these are completed, the status of the drop in $C_{N N}\left(90^{\circ}\right)$ should also be considerably clarified.

An increase in the strength of a spin singlet wave is compatible with the decrease in $C_{N N}$ shown in Fig. 10. This behavior is expected in some quark models, where the lowest lying $\mathrm{I}=1$ dibaryon state is expected to be in the ${ }^{1} S_{0}$ partial wave. In particular, the cloudy bag model of Lomon et al. [58] suggests that a ${ }^{1} S_{0}$ dibaryon state should occur near $\sqrt{s}=2.7 \mathrm{GeV}$. On the other hand, if no dibaryons are present in the Saclay energy range, this would call into question the assumptions that went into the quark models in [5561]. In particular, there may be serious implications for the understanding of nuclear matter.

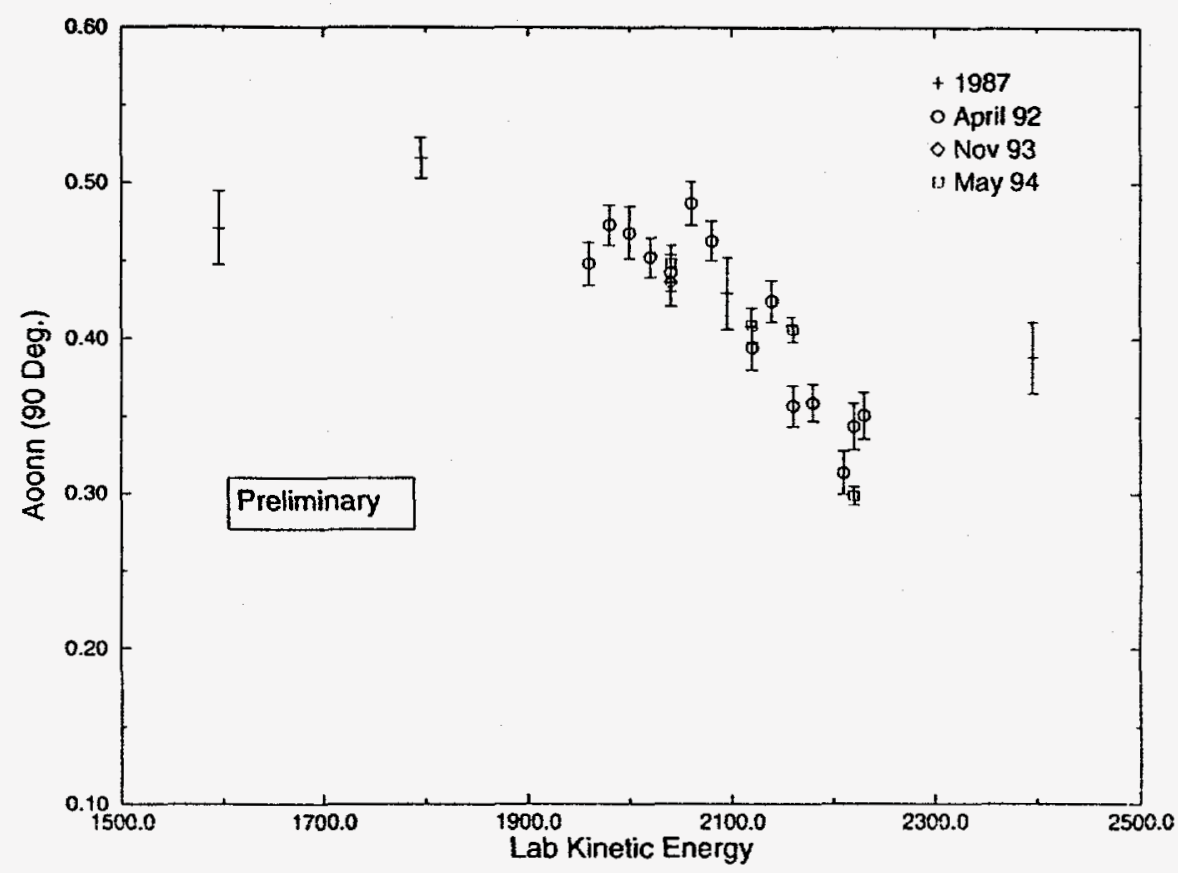

FIGURE 10. Measured values of $C_{N N}\left(90^{\circ}\right)$ data for pp elastic scattering as a function of lab energy. The Nov., 1993 and May, 1994 results are preliminary.

\section{Acknowledgements}

I wish to express my gratitude to my colleagues in the LAMPF and Saclay N-N collaborations and at Argonne National Laboratory for helpful suggestions during the course of this work. I am also thankful for help with some of the figures by C. Allgower, M. Beddo and D. Lopiano. This work was supported in part by the U.S. Department of Energy, Division of High Energy Physics, Contract W-31-109-ENG-38. 


\section{References}

1. For example, see S.J. Wallace, Ann. Rev. Nucl. Sci. 37, 267 (1987) and the talk of $\mathrm{T}$. Taddeucci at this conference.

2. J. Bystricky et al., J. Phys. (Paris) 48, 199 (1987); 51, 2747 (1990); and F. Lehar et al., ibid. 48, 1273 (1987);.

3. D.V. Bugg, Phys. Rev. C41, 2708 (1990).

4. Y. Higuchi et al., Prog. Theor. Phys. 86, 17 (1991); N. Hoshizaki and T. Watanabe, ibid. 86, 321, 327 (1991); Y. Higuchi et al., ibid. 88, 1019 (1992).

5. R.A. Arndt et al., Phys. Rev. D45, 3995 (1992).

6. C.E. Waltham et al., Nucl. Phys. A433, 649 (1985).

7. A.B. Wicklund et al., Phys. Rev. D35, 2670 (1987).

8. R.L. Shypit et al., Phys. Rev. Lett. 60, 901 (1988); 61, 2385 (1988); Phys. Rev. C40, 2203 (1989).

9. I.I. Strakovskii et al., Yad. Fiz. 40, 429 (1984) [trans. Sov. J. Nucl. Phys. 40, 273 (1984)].

10. M.G. Ryskin and I.I. Strakovsky, Phys. Rev. Lett. 61, 2384 (1988).

11. T.-S.H. Lee, Phys. Rev. C40, 2911 (1989).

12. N. Hiroshige et al., Mod. Phys. Lett. A5, 207 (1990).

13. N. Hoshizaki, Phys. Rev. C45, 1424 (1992); Prog. Theor. Phys. 89, 245, 251, 563, 569 (1993).

14. E. Aprile et al., Phys. Rev. Lett. 46, 1047 (1981); R. Hausammann et al., Phys. Rev. D40, 22 (1989).

15. M.J. Moravcsik et al., Phys. Rev. D31, 1577 (1985).

16. M.W. McNaughton et al., Phys. Rev. C41, 2809 (1990).

17. C.D. Lac et al., J. Phys. (Paris) 51, 2689 (1990).

18. I.P. Auer et al., Phys. Rev. D32, 1609 (1985).

19. M. Matsuda et al., Phys. Rev. D33, 2563 (1986).

20. J. Bystricky et al., J. Phys. (Paris) 39, 1 (1978).

21. C. Lechanoine-Leluc et al., J. Phys. (Paris) 48, 985 (1987).

22. R.A. Arndt, Phys. Rev. D37, 2665 (1988); R.A. Arndt et al., ibid. D35, 128 (1987).

23. B.J. VerWest and R.A. Arndt, Phys. Rev. C25, 1979 (1982).

24. J. Bystricky et al., J. Phys. (Paris) 48, 1901 (1987).

25. R.D. Ransome et al., Nucl. Instrum. Meth. 201, 309, 315 (1982); M.W. McNaughton et al., ibid. A241, 435 (1985).

26. R. Garnett et al., Nucl. Instrum. Meth. A309, 508 (1991).

27. M.W. McNaughton et al., Phys. Rev. C44, 2267 (1991).

28. K.H. McNaughton et al., Phys. Rev. C46, 47 (1992).

29. M.W. McNaughton et al., Phys. Rev. C48, 256 (1993).

30. M.W. McNaughton et al., Phys. Rev. C45, 2564 (1992).

31. W.R. Ditzler et al., Phys. Rev. D46, 2792 (1992).

32. T. Shima et al., Phys. Rev. D47, 29 (1993).

33. M. Beddo et al., Phys. Lett. B258, 24 (1991); Phys. Rev. D50, 104 (1994). 
34. J. Ball et al., Nucl. Instrum. Meth. A327, 308 (1993).

35. J. Ball et al., Nucl. Phys. A559, 477 (1993).

36. J. Ball et al., Nucl. Phys. A559, 489 (1993).

37. J. Ball et al., Nucl. Phys. A559, 511 (1993).

38. J. Ball et al., Z. Phys. C61, 579 (1994).

39. J. Ball et al., Nucl. Phys. A574, 697 (1994).

40. C. Lechanoine-LeLuc and F. Lehar, Rev. Mod. Phys. 65, 47 (1993).

41. J.M. Fontaine et al., Nucl. Phys. B358, 297 (1991).

42. J. Ball et al., Z. Phys. C61, 53 (1994).

43. R. Binz et al., Nucl. Phys. A533, 601 (1991).

44. Talk by C.A. Davis at this conference.

45. Talk by B. Vuaridel at this conference.

46. Talk by $\mathrm{S}$. Bowyer at this conference.

47. R.J. Oakes, Phys. Rev. 131, 2239 (1963).

48. F.J. Dyson and N.-H. Xuong, Phys. Rev. Lett. 13, 815 (1964).

49. L.M. Libby, Phys. Lett. 29B, 345 (1969).

50. S. Graffi et al., Lett. Nuo. Cim. 2, 311 (1969).

51. R.F. George et al., Phys. Rev. Lett. 15, 214 (1965); D.V. Bugg et al., Phys. Rev. 146, 980 (1966).

52. R.C. Kammerud et al., Phys. Rev. D4, 1309 (1971).

53. L.M. Libby and E. Predazzi, Lett. Nuo. Cim. 2, 881 (1969).

54. For example, see C. Schmid, Phys. Rev. Lett. 20, 689 (1968).

55. A.Th.M. Aerts et al., Phys. Rev. D17, 260 (1978).

56. C.W. Wong and K.F. Liu, Phys. Rev. Lett. 41, 82 (1978).

57. T. Goldman et al., Phys. Rev. C39, 1889 (1989).

58. P. LaFrance and E.L. Lomon, Phys. Rev. D34, 1341 (1986); P. González and E.L. Lomon, ibid. D34, 1351 (1986); P. González, P. LaFrance, and E.L. Lomon, ibid. D35, 2142 (1987).

59. C.W. Wong, Prog. Part. Nucl. Phys. 8, 223 (1982).

60. Yu.S. Kalashnikova et al., Yad. Fiz. 46, 1181 (1987) [trans. Sov. J. Nucl. Phys. 46, 689 (1987)].

61. N. Konno et al., Phys. Rev. D35, 239 (1987).

62. R. Bertini et al., Phys. Lett. 162B, 77 (1985).

63. R. Bertini et al., Phys. Lett. 203B, 18 (1988).

64. F. Perrot et al., Nucl. Phys. B296, 527 (1988).

65. J.M. Fontaine et al., Nucl. Phys. B321, 299 (1989).

66. I.P. Auer et al., Phys. Rev. Lett. 51, 1411, 1814(E) (1983).

67. I.P. Auer et al., Phys. Rev. D34, 2581 (1986).

68. I.P. Auer et al., Phys. Rev. Lett. 62, 2649 (1989).

69. J. Ball et al., Phys. Lett. B320, 206 (1994).

70. F. Lehar et al., Nucl. Phys. B294, 1013 (1987).

71. D. Miller et al., Phys. Rev. D16, 2016 (1977).

72. A. Lin et al., Phys. Lett. 74B, 273 (1978). 